A flexible method to measure synchrony in neuronal firing

Peer-reviewed author version

FAES, Christel; GEYS, Helena; MOLENBERGHS, Geert; AERTS, Marc;

Cadarso-Suarez, Carmen; Acuna, Carlos \& Cano, Monica (2008) A flexible method to measure synchrony in neuronal firing. In: JOURNAL OF THE AMERICAN

STATISTICAL ASSOCIATION, 103(481). p. 149-161.

DOI: $10.1198 / 016214507000000419$

Handle: http://hdl.handle.net/1942/8179 


\title{
A Flexible Method to Measure Synchrony in
}

\section{Neuronal Firing.}

\author{
Christel Faes, Helena Geys, Geert Molenberghs, Marc Aerts, \\ Carmen Cadarso-Suárez, Carlos Acuña and Mónica Cano *
}

\begin{abstract}
Neurons can transmit information about the characteristics of a stimulus via the spike rate of neurons and via synchronization of the neurons. To describe how 'synchronous' two spike trains are, a variety of association measures can be used. We propose a new measure of synchrony, the conditional synchrony measure, which is the probability of firing together given that at least one of the two neurons is active. Focus is on the specification of a flexible marginal model for multivariate correlated binary data together with a pseudo-likelihood estimation approach, to adequately and directly describe the measures of interest. A joint model must allow different time- and covariatedepending firing rates for each neuron, and must account for the association between them. The association between neurons might depend on covariates as well.
\end{abstract}

${ }^{*}$ Christel Faes, Helena Geys, Geert Molenberghs, Marc Aerts, Center for Statistics, Hasselt University, Diepenbeek, Belgium (email: christel.faes@uhasselt.be). They gratefully acknowledge support from the Institute for the Promotion of Innovation by Science and Technology in Flanders, from the Fund for Scientific Research Flanders and from the IAP research network nr P5/24 of the Belgian Government. Carmen Cadarso-Suárez, Department of Statistics and Operations Research, University of Santiago de Compostela, Spain. Carlos Acuña, Department of Physiology, University of Santiago de Compostela, Spain. Their work was funded by grants BMF2002-03213, PM99-0025 and 2003/PC014 from the Spanish Ministry of Science and Technology. Mónica Cano, Department of Psychology, University of Connecticut, USA. We thank the anonymous referees and associate editor for the many suggestions that have helped in improving the quality of this paper. 
Keywords: Conditional Synchrony Measure, Neural Synchrony, Neurophysiology, Pseudo-Likelihood Methodology

\section{Introduction}

Neurons in the nervous system transmit information by sequences of action potentials, but the neural code used is a matter of debate. Firing rate and temporal correlation are two of the codes the nervous system can use to transmit information (Shadlen and Newsome 1994, Eggermont 1998). The important characteristic of a train of spikes is the mean firing rate; this implies that the parameters of the stimulus can be coded by adjusting the firing rate to some parameter of the stimulus, and the neural response would be de-codified by counting the action potentials (Eggermont 1998). There is evidence for association between spike rate and psychophysical performance (Mountcastle et al. 1990, Newsome et al. 1989, Romo and Salinas 1999). Alternatively, neurons can behave as coincidence detectors; they can only fire if a reduced number of excitatory events arrive simultaneously in a temporal scale of milliseconds (Abeles 1982, Konig et al. 1996). These temporal coincidences are called synchronized activity and it is hypothesized that it should be correlated to perceptual grouping of feature (Malsburg 1999, Usrey and Reid 1999, Shadlen and Movshon 1999, Abeles et al. 1994).

The aim of this work is to develop a statistical method of analysis which allows us to study synchrony between simultaneously recorded neurons under a variety of conditions. Furthermore, new electrophysiological methodology allows recording multiple neural data simultaneously. The challenge is to developed statistical tools to study this simultaneous recorded neural activity (Brown et al. 2005). The approach presented here allows this analysis. The orientation of a line in visual space is represented by neurons in an orientation column of the primary visual cortex (V1), i.e., a small volume of visual cortex (Hubel and Wiesel 1969). Neurons in a given column prefer lines with a certain orientation (i.e., orientation selectivity). Preferred 
orientation refers to the maximal firing rate of the neuron provoked by some line orientation. Columns of preferred orientation systematically change as we move across the visual space (Hubel et al. 1978). A long line with a particular orientation which falls on several receptive fields, each with different orientation, would produce individual neural responses of different strengths. How does the visual system recognize it as a single line even though the response of each neuron is different? The neural mechanisms underlying perceptual grouping may use synchrony, and this hypothesis suggests that neurons responding to features belonging to the same perceptual group are synchronized such that they fire together (Singer and Gray 1995, Shadlen and Movshon 1999, Gray et al. 1989). Therefore, as a first step, we set out to asses synchrony between simultaneously registered activities of pairs of neurons in V1 associated with changes in the orientation of lines placed on the neurons receptive fields during performance of a discrimination task. The use of the discrimination task guarantees subjects' active perception of the orientation of a line. Our main goal is to validate our statistical approach. Later, we can use the proposed methods to asses the influence of other behavioral variables, such as eye movements.

Nowadays the study of multiple neural spike trains and synchrony are still in development and constructing new multivariate statistical models to solve the problem are strongly encouraged by the physiologists' community (Brown et al. 2005). Conventional approaches are based on the use of cross-correlation techniques, usually applied to the activity of pairs of neurons recorded under appropriate stimulus conditions. In this context, the basic tool is the cross-correlogram, representing a time-averaged correlation among the spiking events of the participating neurons. Extensions of this analysis are the Gravitational Clustering (Gerstein and Aertsen 1985) and the Joint-Peristimulus Time Histogram (Aertsen et al. 1989), which address the dynamics of the correlation between cells on a very short time scale. However, although the dynamics of synchronicity can be observed as a function of time by averaging over trials, with these approaches it is not possible to analyze 
individual spike coincidences on a trial-by-trial basis.

Recent investigations have focused on the detection of individual instances of synchronized activity between groups of two or more neurons. One of the most commonly used methods for this task is the Unitary-Event (UE) analysis (Grün 1996, Grün et al. 1999, Grün, Diesmann, and Aertsen 2002a, 2000b, Riehle et al. 1997). This approach allows us to ascertain the statistical significance of brief epochs of synchronous spiking. The statistical null-hypothesis is formulated in terms of the individual firing probabilities of the participating neurons. This method searches recordings from multiple single neurons for epochs with distinctly more (approximately-)coincident spikes than expected from independent neurons obeying Poissonian spike statistics. The core of UE analysis consists of computing the probabilities (joint $p$-values) for the occurrence of a given minimum number of coincident spikes in short time segments, under the null hypothesis of independence. Segments with a joint $p$-value below a fixed level of significance are identified as significant epochs where the null hypothesis is rejected. Gütig, Aertsen and Rotter (2002) reformulated the statistical test underlying this method using a coincidence count distribution based on empirical spike counts rather than on estimated spike probabilities. Recently, Kass, Ventura and Cai (2003) considered a more general framework that could handle problems of alternative structures avoiding the assumption that spike trains are Poisson processes and suggest a (simulation-based) significance test for synchrony, in which $p$-values are calculated by using bootstrap techniques.

Alternative procedures to assess synchrony were suggested recently and lie within the general regression framework. Models for multivariate correlated binary data can be grouped into different classes along the distinction between conditionally specified models, marginal models and cluster-specific models. The answer to the question which model family is to be preferred principally depends on the research question(s) to be answered. Conditional models describe the distribution of the outcomes conditional on (a subset of) the other outcomes. Well-known members 
of this class of models are log-linear models. Martignon et al. (2000) introduced a log-linear model for representing firing rates on a set of neurons and showed that nonzero coefficients or effects of these models are a natural measure for synchronous firing. Advantages of the log-linear model are that it provides a relatively simple representation of association: the main effect terms may be taken to be smooth functions of time using regression splines and then the model may be fitted by using standard software for generalized linear models. In this way, standard likelihoodbased estimation and testing of the interaction coefficients provide an assessment for association. Generalization to more than two neurons involving two-way interactions is easy. Higher order interactions may also be examined, and in a sense this is an attractive feature of the approach. On the other hand there are obvious complexities in including large numbers of terms. Another disadvantage of this approach is that the main effect terms are not interpretable as marginal firing rates. Indeed, as many authors have pointed out (e.g., McCullagh and Nelder 1989) the lack of compatibility between marginal models and joint models is a general feature of loglinear models.

So far, non of the existing methods included trial-specific covariates to assess their possible effect in the individual firing rates of the neurons involved or in their synchrony. In this paper, we will consider an alternative approach based on a marginal model for multivariate correlated binary data. This model was designed to (i) describe the individual activity of the neurons involved and (ii) detect correlations of any order in a unified way. This method allows to jointly analyze the firing rates of the individual neurons as well as the synchrony between the neurons. Both the temporal evolution, as well as trial-specific covariates, can be investigated in a flexible way. In addition, the extension to more than two neurons is also possible.

The paper is structured as follows. Section 2 introduces the electrophysiological experiment. In Section 3 we describe some measures of synchrony that are used in the literature and offer a new methodology to measure the neuronal synchrony. Section 4 discusses specific and general issues in modelling the data. In Section 5, 


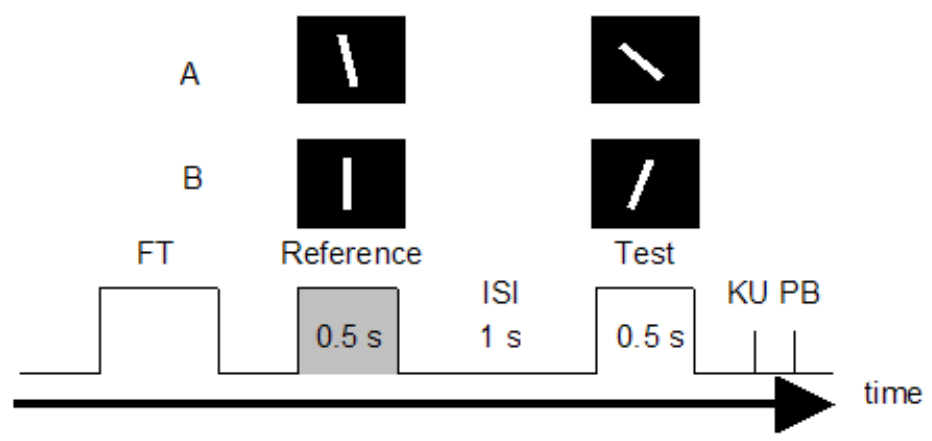

Figure 1: Temporal sequence of the discrimination task.

specific tools for analysis will be exemplified.

\section{The Experiment}

Experiments were carried out on a male monkey (Macaca mulatta). The experimental set-up, description of the stimuli, and behavioural tasks are described in detail elsewhere (Vazquez et al. 2000). The monkey looks binocularly at a monitor screen placed at $114 \mathrm{~cm}$ from their eyes. He has his head fixed during the task, and his right arm operated a lever. A panel with three buttons was in front of the monkey within hand reach. Right and left buttons were used in the discrimination tasks to signal the orientation of the visual stimuli to the right and to the left, respectively. The monkey used the third button in the eye fixation task to signal the tilt of the fixation bar. The eye fixation task is use to calibrate the set up and to map the visual receptive fields. The stimuli were stationary bright lines. Three different reference orientations were used: $85^{\circ}, 90^{\circ}$, and $95^{\circ}$. Different test lines, 8 per reference stimulus, were presented clockwise and counter-clockwise to the reference line in steps of $1^{\circ}$. The stimuli, oriented lines, were presented in the center of the monitor screen. During the trial, This is an area of current interest for the authors eye movements larger than $2.5^{\circ}$ aborted the task. A masking white 
noise signaled the beginning of the trial, and was initiated with the presentation of the fixation target when the monkey pressed the lever key with his free hand (FT, Figure 1). The FT disappears through a variable pre-stimulus delay (600-900 ms), and then, two stimuli (reference and test), each of 500 ms duration, were presented in sequence, with a fixed interstimulus interval (ISI, 1s). At the end of the second stimulus, the subject released the key (KU) and pressed one of the two buttons, indicating whether the orientation of the second stimulus was clockwise or counterclockwise to the first (PB). Monkeys were rewarded with a drop of liquid for correct discriminations.

The activity of neurons in the primary visual cortex were recorded simultaneously, through the insertion of different micro-electrodes into the monkey's brain, while the monkey performed the visual discrimination task. To study the synchrony between cell pairs the analysis was restricted to the effect of the three reference stimuli (500 $\mathrm{ms})$. The $200 \mathrm{~ms}$ before the reference stimulus comes on can be taken as control (baseline cell activity), due to the fact that during this period there was no visual stimulus whatsoever. Another period of $200 \mathrm{~ms}$ out of the $1000 \mathrm{~ms}$ of the InterStimulus Interval (ISI) was also taken as control, i.e., recovery of the cell activity. We emphasize the fact that subjects can only solve the discrimination task by paying attention to both stimuli in each trial. In fact, when in the discrimination task the first stimulus is not shown, humans and monkeys cannot solve the task (Hernandez et al. 1997, Vazquez et al. 2000). This indicates that subjects cannot anticipate the orientation of the stimulus. Per trial, every action potential is recorded during these $900 \mathrm{~ms}$, resulting in 900 binary outcomes. We examine data from 19 trials, with stimuli at different orientations.

Figure 2 shows the raster plots and peri-stimulus time histograms (PSTHs) of the neuron 1 (top) and neuron 2 (bottom) simultaneously recorded through two independent electrodes 305 microns apart while the monkey performs the discrimination task. On the time axes, $0 \mathrm{~ms}$ corresponds to the appearance of the stimulus and $500 \mathrm{~ms}$ to the removal of the stimulus. The raster plot shows the spike trains 

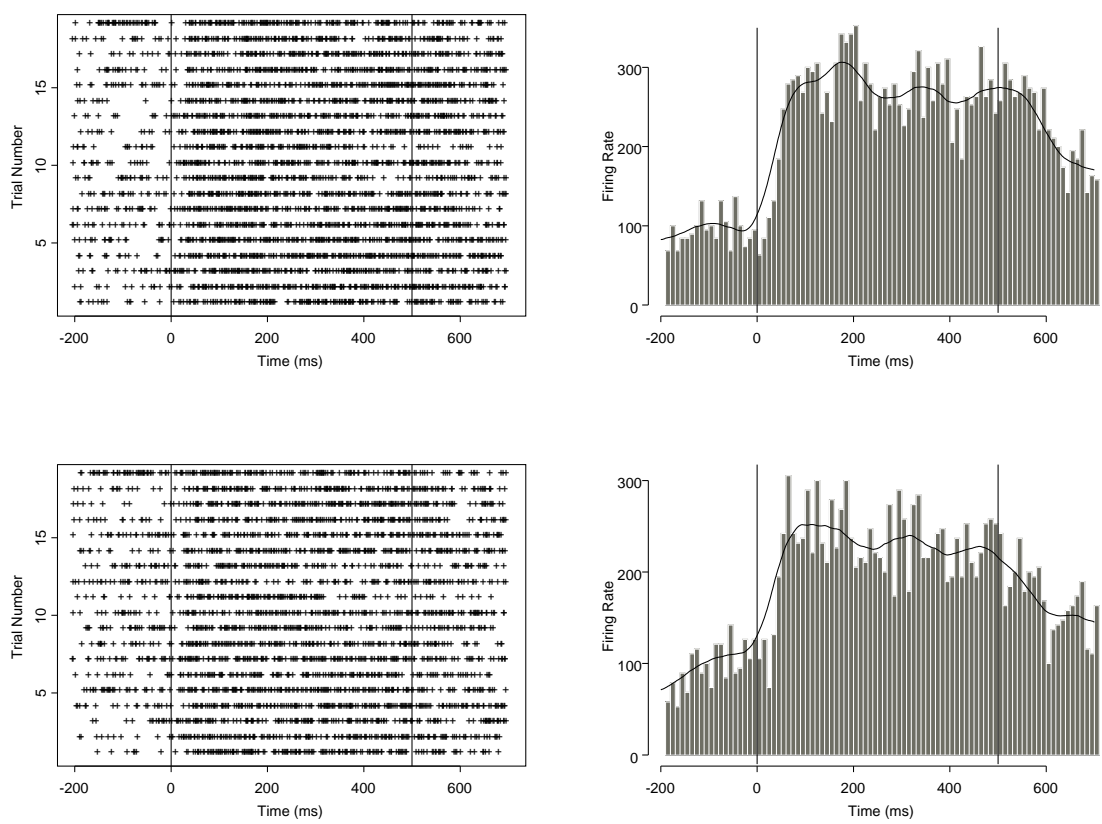

Figure 2: Raster plot (left) of spikes and corresponding peristimulus time histogram (right) with smoothed (kernel) version of the counts for neuron 1 (top) and neuron 2 (bottom).

for each of the trials on separate lines. Trial numbers 1 to 7 correspond with a reference line of $85^{\circ}$, trial numbers 8 to 14 with an orientation of $90^{\circ}$, and in trials 15 to 19 a reference line of $85^{\circ}$ was shown. The peristimulus time histogram (PSTH) displays the firing rate, i.e., the number of spikes per second. The firing rate is calculated in $10 \mathrm{~ms}$ bins and averaged over all trials. The solid curve is a smoothed version of the counts obtained with a kernel density estimator. After the appearance of the stimulus, the intensity of spikes increases rapidly up to some maximum, and then remains high. After removal of the stimulus, the intensity of spikes gradually drops back. The intensity of spikes in the first neuron is higher than in the second neuron. 
Table 1: A contingency table of the number of matches and mismatches for two neurons at a certain time point.

\begin{tabular}{lr|cc|c} 
& & \multicolumn{2}{|c|}{ Neuron $k$} & \\
& & 1 & 0 & Totals \\
\hline \multirow{2}{*}{ Neuron $i$} & 1 & $n_{11}$ & $n_{10}$ & $n_{11}+n_{10}$ \\
& 0 & $n_{01}$ & $n_{00}$ & $n_{01}+n_{00}$ \\
\hline Totals & $n_{11}+n_{01}$ & $n_{10}+n_{00}$ & $N=n_{11}+n_{10}+n_{01}+n_{00}$
\end{tabular}

\section{Measures of Synchrony}

Synchrony refers to the observation that action potentials emitted from different neurons are emitted at the same time, or very close in time. In this paper, two neurons are considered to discharge in synchrony if they fire together in a $1 \mathrm{~ms}$ window. However, methods can easily be extended to less precise coincidences. First, focus is on the synchrony of two neurons only. Later, an extension of the methodology to more than two neurons is proposed. To describe how 'synchronous' two spike trains are, a variety of methods can be used. Let us first introduce the necessary notation.

Let $Y_{i t}$ be the binary outcome of the $i$ th neuron at time $t$, for $t=1,2, \ldots, T$. The frequency of matches and mismatches over the different trials for neurons $i$ and $k$ at time point $t$ can be written in the form of a contingency table, such as displayed in Table 1. In this table, $n_{11}$ represents the frequency of $1-1$ matches (at time $t$ ), $n_{10}$ is the frequency of $1-0$ matches, and so forth. The matching rate for neuron 1 and neuron 2 at different time points are pictured in Figure 3. The probability of coincidence increases rapidly after appearance of the stimulus and decreases gradually after removal of the stimulus. Note however that one has to be careful in interpreting the increase in the number of synchronous events, since a certain number of synchronous events would always occur due to purely 

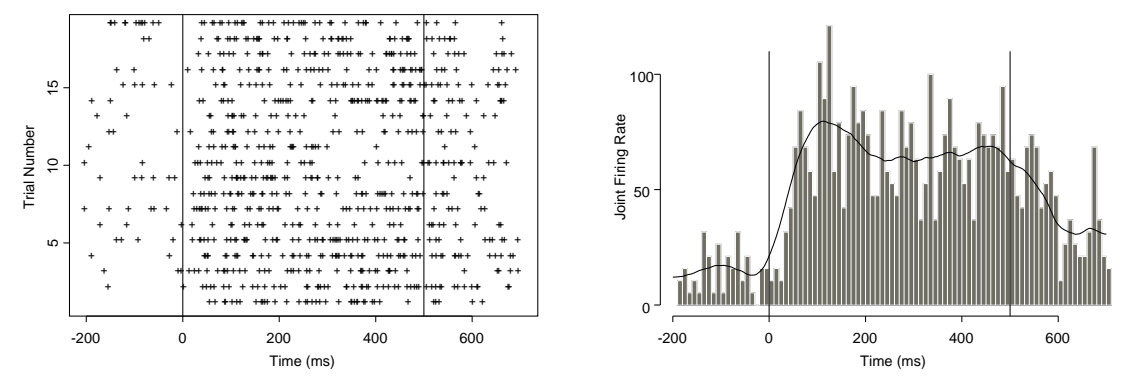

Figure 3: Raster plot (left) of spike coincidence of neuron 1 and 2, and corresponding joint peristimulus time histogram with smoothed (kernel) version of the counts (right).

random coincidence. In the next section, we give an overview of possible synchrony measures.

\subsection{Odds Ratio}

One possible measure for synchrony is the odds ratio, indicating the association between two neurons. The odds ratio is often a measure of choice to capture the association in a contingency table. The odds ratio at time $t$ is defined as

$$
\psi(t)=\frac{n_{11} n_{00}}{n_{10} n_{01}}=\frac{\pi_{11}(t)\left[1-\pi_{1+}(t)-\pi_{+1}(t)+\pi_{11}(t)\right]}{\left[\pi_{11}(t)-\pi_{1+}(t)\right]\left[\pi_{11}(t)-\pi_{+1}(t)\right]},
$$

with $\pi_{11}($.$) the joint probability of 2$ neurons to fire and $\pi_{1+}($.$) and \pi_{+1}($.$) the$ marginal probabilities to fire. Using this relationship, the joint distribution of neuron 1 and 2 can be written as function of the marginal probabilities and the odds ratio (Plackett 1965):

$$
\pi_{11}(t)= \begin{cases}\frac{1+\left[\pi_{1+}(t)+\pi_{+1}(t)\right][\psi(t)-1]-R\left(\pi_{1+}(t), \pi_{+1}(t), \psi(t)\right)}{2[\psi(t)-1]}, & \text { if } \psi \neq 1 \\ \pi_{1+}(t) \pi_{+1}(t), & \text { otherwise }\end{cases}
$$

with

$$
R\left(\pi_{1+}, \pi_{+1}, \psi\right)=\sqrt{\left[1+\left(\pi_{1+}+\pi_{+1}\right)(\psi-1)\right]^{2}+4 \psi(1-\psi) \pi_{1+} \pi_{+1}} .
$$



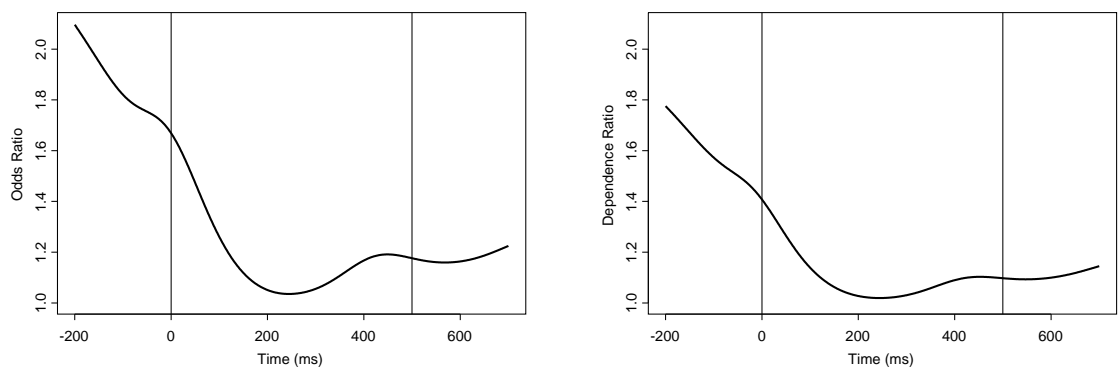

Figure 4: Plot of odds ratio (left panel) and dependence ratio (right panel) between neuron 1 and neuron 2.

The Plackett-Dale distribution has been used extensively in the modelling of many biomedical applications. For example, it turned out to be very useful in the modelling of clustered binary data together with a pseudo-likelihood estimation approach (Geys, Molenberghs, and Lipsitz 1998).

A plot of the odds ratio as function of time between neuron 1 and 2 is given in the left panel of Figure 4. All values are higher than 1, indicating a positive association between the two neurons. But, in contrast with the probability of joint firing, there is a decrease at the start of the stimulus, and a small increase at the end of the stimulus. The elevated odds ratios before the appearance of the stimulus might be explained by the low activity rate, or high number of $0-0$ matches, in this time interval (Figure 2). Note however that synchrony is a process defined as happening only during activity of the neurons. Thus, although the odds ratio is an attractive association measure, with nice mathematical properties, such as the absence of range restrictions, regardless of the marginal probabilities, it is less suitable to quantify synchrony due to its symmetry, treating $0-0$ matches of equal importance as $1-1$ matches. Ideally, the fact that a $0-0$ match is a less strong, or even no, indication for synchrony should be reflected in the measure to be used. 


\subsection{Dependence Ratio}

As an alternative, synchrony can be measured as the proportion of observed number of synchronous events to the expected number from two independent stochastic processes. This measure expresses to which extent the probability of having a spike simultaneously for two neurons is different from the product of the marginal probabilities. This idea was exploited by several authors (Gütig, Aertsen and Rotter 2002, Grün et al. 1999, Riehle et al. 1997).

Based on the same idea, Ekholm (1995) defined the dependence ratio as

$$
\tau(t)=\frac{\pi_{11}(t)}{\pi_{1+}(t) \pi_{+1}(t)} .
$$

It is easy to see that $(\tau-1) \times 100$ indicates the increase (as \%) in probability for both neuron 1 and neuron 2 to have a spike, compared to what it would be under independence; that $\tau=1$ if and only if neurons 1 and 2 are independent; and that the following relationship between $\tau$ and $\psi$ holds:

$$
\psi=\frac{\tau-1}{\left(1-\tau \pi_{1+}\right)\left(1-\tau \pi_{+1}\right)}+1
$$

In Figure 4, the dependence ratio between neurons 1 and 2 is depicted. The $\tau$-curve is almost identical to the plot of the odds ratio. There is a downwards shift of about 0.2 . Similar to the odds ratio, it seems that the dependence ratio is highly affected by the small number of spikes at the start and end of the experiment. Further, note that when neurons are dependent, they are not necessarily in synchrony. For example, consider two neurons with the following spikes during several trials:

neuron 1: 110011101001,

neuron 2: 001100010110.

These neurons are asynchronous (none of the events collapse), although the neurons are dependent $\left(\pi_{11} \neq \pi_{1+} \pi_{+1}\right)$. Thus, one should be cautious with the use of independence as a basis for a measure of synchrony. 
Table 2: Some Possible Similarity Measures in Terms of Frequencies.

\begin{tabular}{|c|c|}
\hline Measure & Rationale \\
\hline$\frac{n_{11}}{n_{11}+n_{10}+n_{01}}$ & $\begin{array}{l}\text { No } 0-0 \text { matches in numerator or denominator. The } 0-0 \text { matches } \\
\text { are treated as irrelevant. }\end{array}$ \\
\hline$\frac{2 n_{11}}{2 n_{11}+n_{10}+n_{01}}$ & $\begin{array}{l}\text { No } 0-0 \text { matches in numerator or denominator. Double weight } \\
\text { for } 1-1 \text { matches. }\end{array}$ \\
\hline$\frac{n_{11}}{n_{11}+2\left(n_{10}+n_{01}\right)}$ & $\begin{array}{l}\text { No } 0-0 \text { matches in numerator or denominator. Double weight } \\
\text { for unmatched pairs. }\end{array}$ \\
\hline$\frac{n_{11}}{0+n_{01}}$ & Ratio of matches to mismatches with $0-0$ matches excluded. \\
\hline
\end{tabular}

\subsection{Conditional Synchrony Measure}

A measure of synchrony is to be regarded as a specific measure of 'closeness', or 'similarity', and should treat a $1-1$ match as a stronger indication of similarity than a $0-0$ match. Indeed, the evidence that two neurons react is stronger evidence of synchrony than the absence of a spike in both neurons. Thus, it might be reasonable to discount the $0-0$ matches or even disregard them entirely. Table 2 lists some similarity measures in terms of the frequencies. The first three measures are monotonically related (Johnson and Wichern 1989, p. 732-733). The different measures of similarity are pictured in Figure 5. These plots all give the same idea of synchrony. After the stimulus is given, there is an increase in synchrony. After the end of the stimulus, there is a decrease.

One possible appealing measure of synchrony, which could be used in the context of neuronal synchrony, is the first one from Table 2. The main advantage is the absence of the nuisance $(0,0)$ pairs. The conditional probability of firing together, given there is a spike in one of the neurons, could be used to measure the 'strength' of synchrony. This measure, which we call the Conditional Synchrony Measure 

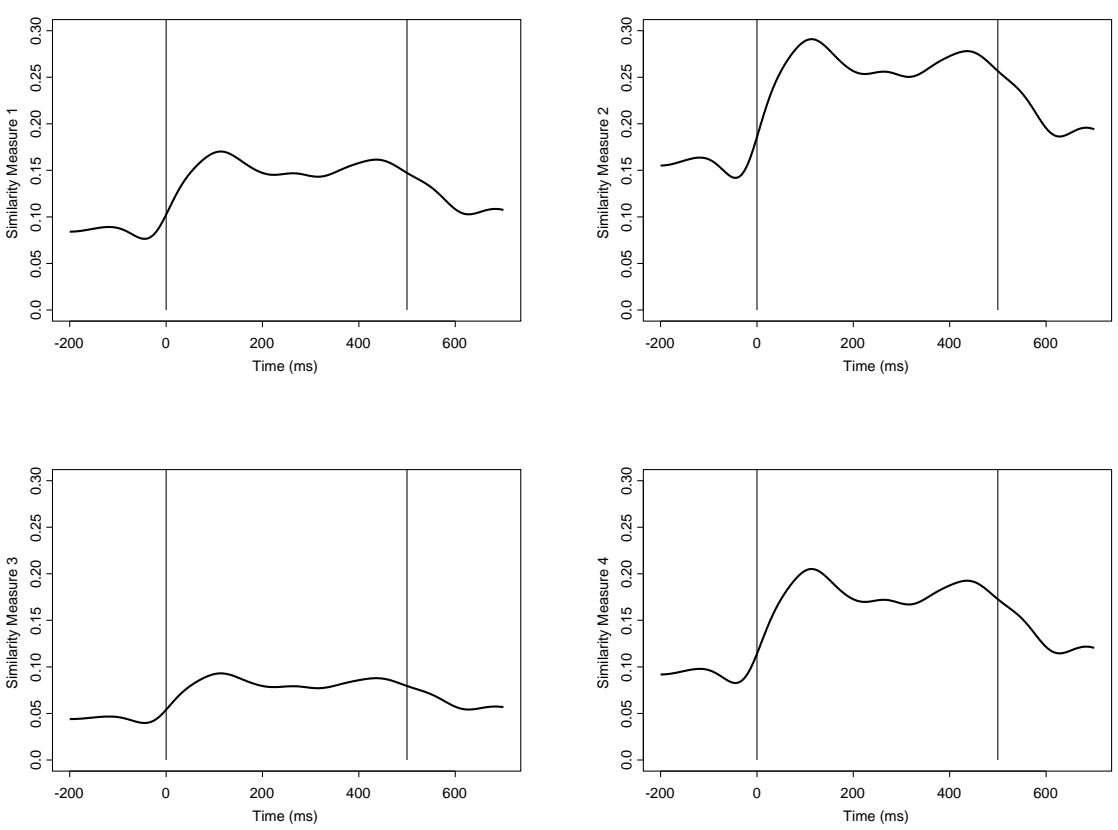

Figure 5: From left to right and from top to bottom: a plot of similarity measures proposed in Table 1, for neuron 1 and neuron 2.

(CSM), reflects how much the neurons fire together during activity in one of the two neurons. It can be written as a function of the joint probability of firing and the two marginal probabilities:

$$
\operatorname{CSM}(t)=\frac{\pi_{11}(t)}{\pi_{1+}(t)+\pi_{+1}(t)-\pi_{11}(t)}
$$

estimated by

$$
\widehat{\operatorname{CSM}}(t)=\frac{n_{11}}{n_{10}+n_{01}+n_{11}}
$$

The CSM has an easy to understand interpretation, and is based on the definition of synchrony. The top left panel in Figure 5 shows a plot of the CSM for neurons 1 and 2. This plot shows an increase of synchrony after the stimulus, and a decrease of synchrony after the end of stimulus.

In what follows, we will use the CSM as a measure of synchrony. A few remarks 
are in place. First, by developing this measure further, we do not claim it is the only one of interest, and other choices from Table 2 are worthy of exploration as well. However, due to the monotonic relationship between the first three, it is sufficient to consider a single one. Second, the asymmetry comes at the price of range dependence of the CSM on the marginal probabilities, unlike the odds ratio. Note, however, that even the Pearson correlation coefficient in the case of two binary outcomes cannot attain its full range $[-1,1]$. In Section 4, a model based on the conditional probability is described, and is combined with a pseudo-likelihood estimation method. Section 5 describes the analysis of the experiment.

\section{Model Formulation and Pseudo-likelihood}

\subsection{The Model}

Suppose that for each neuron $i(i=1,2)$ under study a set of binary responses $\left\{Y_{i t}, t=1, \ldots, T\right\}$ is observed, together with a vector of covariates $\boldsymbol{x}$. Assume that observations at different time points are independent for now. Later, in Section 4.2, their dependence will be incorporated in the analysis. We want to establish the dependence of each of the two neurons on the covariate vectors, taking the correlation between both responses into account.

The model arises from the decomposition of the joint probabilities

$$
\pi_{j_{1} j_{2}}(t)=P\left(Y_{1 t}=j_{1}, Y_{2 t}=j_{2} \mid \boldsymbol{x}\right), \quad\left(j_{1}, j_{2}=0,1\right),
$$

into 'main effects' and 'effect of synchrony'. Let the marginal probabilities of neurons 1 and 2 at time $t$ be $\pi_{1+}(t)$ and $\pi_{+1}(t)$, respectively. The Conditional Synchrony Measure, or conditional probability of observing two spikes at time $t$, given there is a spike in at least one of the two neurons, as defined in (3.6). Formally, the 
decomposition of the joint probabilities $\pi_{j_{1} j_{2}}$ is given by

$$
\begin{aligned}
& h_{1}\left(\pi_{1+}(t)\right)=\beta_{1}^{T} \boldsymbol{x}, \\
& h_{2}\left(\pi_{+1}(t)\right)=\beta_{2}^{T} \boldsymbol{x}, \\
& h_{3}(\operatorname{CSM}(t))=\beta_{3}^{T} \boldsymbol{x},
\end{aligned}
$$

with $h_{1}, h_{2}$, and $h_{3}$ monotonic differentiable functions. As such, the marginal structure can be modeled in a flexible fashion: the marginal probabilities can be fitted within the generalized linear models framework. And also the synchrony can be modeled in a general way, including time-varying covariates as well as trial-specific covariates.

Solving these equations for the joint probability $\pi_{j_{1} j_{2}}(t)$ yields:

$$
\pi_{11}(t)=\frac{\operatorname{CSM}(t)}{1+\operatorname{CSM}(t)}\left[\pi_{1+}(t)+\pi_{+1}(t)\right] .
$$

Based upon this probability, we can derive the joint density function

$$
g\left(y_{1 t}, y_{2 t}\right)= \begin{cases}\pi_{11}(t) & \text { if } y_{1}=1 \text { and } y_{2}=1, \\ \pi_{1+}(t)-\pi_{11}(t) & \text { if } y_{1}=1 \text { and } y_{2}=0, \\ \pi_{+1}(t)-\pi_{11}(t) & \text { if } y_{1}=0 \text { and } y_{2}=1, \\ 1-\pi_{1+}(t)-\pi_{+1}(t)+\pi_{11}(t) & \text { if } y_{1}=0 \text { and } y_{2}=0 .\end{cases}
$$

Sometimes, interest is in the synchrony of three or more neurons, or in the probability of joint firing of three or more neurons. The above model can be generalized to model $M$ neurons simultaneously in the presence of explanatory variables $\boldsymbol{x}$. Let $\left\{Y_{i t}, t=1, \ldots, T\right\}$ be the random vector for neuron $i(i=1,2, \ldots, M)$. Let $\pi_{j_{1}, j_{2}, j_{3}}(t)$ be the joint probability

$$
\pi_{j_{1} j_{2} j_{3}}(t)=P\left(Y_{1 t}=j_{1}, Y_{2 t}=j_{2}, Y_{3 t}=j_{3} \mid \boldsymbol{x}\right),
$$

with $j_{1}, j_{2}, j_{3}$ equal to 0 or 1 . The synchrony of 3 neurons can be defined as the conditional probability of joint firing in the three neurons, given there is activity in at least one of the neurons

$$
\operatorname{CSM}_{123}(t)=\frac{\pi_{111}}{\pi_{1++}+\pi_{+1+}+\pi_{++1}-\pi_{11+}-\pi_{1+1}-\pi_{+11}+\pi_{111}}
$$


where all probabilities depend on the time $t$. Using this equation, one can rewrite the joint probability as function of the 'main effects', 'synchrony between two neurons' and 'synchrony between three neurons':

$$
\begin{aligned}
\pi_{j_{1} j_{2} j_{3}=}=\frac{\operatorname{CSM}_{123}}{1-\operatorname{CSM}_{123}}\left[\pi_{1++} \frac{1-\mathrm{CSM}_{12} \mathrm{CSM}_{13}}{\left(1+\mathrm{CSM}_{12}\right)\left(1+\mathrm{CSM}_{13}\right)}\right. \\
\left.\quad+\pi_{+1+} \frac{1-\mathrm{CSM}_{12} \mathrm{CSM}_{23}}{\left(1+\mathrm{CSM}_{12}\right)\left(1+\mathrm{CSM}_{23}\right)}+\pi_{++1} \frac{1-\mathrm{CSM}_{13} \mathrm{CSM}_{23}}{\left(1+\mathrm{CSM}_{13}\right)\left(1+\mathrm{CSM}_{13}\right)}\right] .
\end{aligned}
$$

To simplify notation, we ignored the time-dependency in the above equation. As such, one can jointly investigate the firing rates in the three neurons and test the synchrony between three neurons. A generalization to more than three neurons is also possible.

\subsection{Pseudo-likelihood Estimation Method}

Arguably, observations at different time points are not independent. Three different types of associations, depicted in Figure 6 , can be present: the association between two different time points from the same neuron $(\omega)$, the association between two neurons at the same time point $(\delta)$, and the association between two neurons at two different time points $(\gamma)$. Although there is only one association of direct interest, namely the association between two neurons at the same time point, the other associations cannot be neglected. Indeed, ignoring the associations in the data overestimates precision and hence underestimates standard errors and lengths of confidence intervals. One can treat such associations as a nuisance, correcting for them to obtain suitable variance estimates. In such a case, we can use pseudolikelihood, rather than considering full likelihood. The pseudo-likelihood approach was proposed by Arnold and Strauss (1991), and studied in Connolly and Liang (1988), Liang and Zeger (1986), le Cessie and Van Houwelingen (1994) and Geys, Molenberghs and Ryan (1997). In addition, the pseudo-likelihood method provides a way to deal with nuisance parameters. Details of the pseudo-likelihood approach are given in the appendix. 
neuron 1

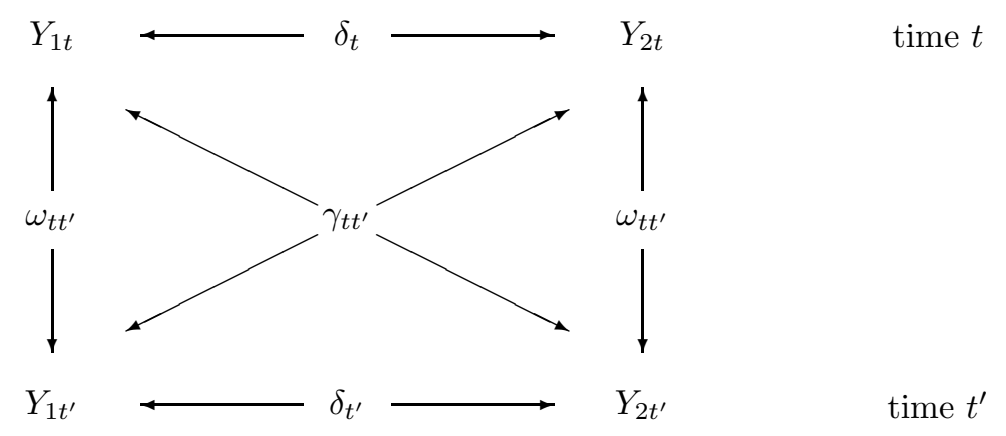

Figure 6: Four different types of contributions for the pseudo-likelihood (for a fixed trial).

The principal idea of the pseudo-likelihood estimation method is to replace the numerically intractable joint density by a simpler function that is a suitable product of ratios of likelihoods of subsets of the variables that do not necessarily multiply to the joint distribution. The full likelihood function for trial $j(j=1, \ldots, N)$, i.e., $f_{j}\left(y_{11 j}, \ldots, y_{1 T j}, y_{21 j}, \ldots, y_{2 T j}\right)$, can be replaced by

$$
p \ell_{j}=\sum_{j=1}^{N} \ln g\left(y_{1 t j}, y_{2 t j}\right),
$$

where the index $j$ indicates the outcomes of trial $j$. As such, we avoid the computational complexity of the full likelihood distribution of each trial. The value of the parameters that maximize the log pseudo-likelihood function $p \ell=\sum_{j=1}^{N} p \ell_{j}$ are the pseudo-likelihood estimates.

Arnold and Strauss (1991) presented a formal and more general definition of the pseudo-likelihood estimation method, and established consistency and asymptotic normality of the pseudo-likelihood estimator. Geys, Molenberghs and Lipsitz 
(1998) compared pairwise likelihood with other estimation equation approaches in marginally specified odds ratio models with exchangeable association structure, and showed that the efficiency of pseudo-likelihood estimators was comparable to the efficiency of GEE estimators. Further, in order to perform a flexible model selection, one would need extensions of the Wald, score or likelihood ratio test statistics to the pseudo-likelihood framework. These were proposed by Geys, Molenberghs and Ryan (1997).

\subsection{Test for Synchrony}

A possible information processing strategy in the nervous system is the use of synchrony between neurons. In this mechanism, the selection of specific sensory information is implemented by increasing the synchrony between neurons that represent the information. Therefore, it is of interest to test whether there is an increase of synchrony during the task.

A sensible approach is to compare the CSM during the experiment with the $\mathrm{CSM}$ at baseline $\left(\mathrm{CSM}^{0}\right)$ :

$$
H_{0}: \operatorname{CSM}(t)=\operatorname{CSM}^{0}
$$

The period before the stimulus comes on (time $-200 \mathrm{~ms}$ until $-1 \mathrm{~ms}$ ) can be used to estimate the baseline cell activity. The condition to be checked is very simple: is the $\widehat{\mathrm{CSM}}(t)$ different from the estimated baseline $\widehat{\mathrm{CSM}}^{0}$.

Often however, one thinks about synchrony in terms of the probability of joint firing. But, as mentioned before, one needs to be careful in interpreting the mechanisms of joint firing. We cannot just compare the joint firing rate with the background firing rate, to test whether there is an increased or decreased synchrony between two neurons. Indeed, an increase of joint firing might be induced by an increase of synchronous action potentials or might be a byproduct of the increased firing rates. Yet, we can translate the above test hypothesis for synchrony in terms of a test on the joint firing probability. Assume the stimulus has no effect on the 
Conditional Synchrony Measure, and is equal to the background $\mathrm{CSM}^{0}$. Then, the probability of joint firing at time $t$, under the null hypothesis of a constant CSM, equals

$$
\pi_{11}^{H_{0}}(t)=\frac{\operatorname{CSM}^{0}}{1+\operatorname{CSM}^{0}}\left[\pi_{1+}(t)+\pi_{+1}(t)\right]
$$

By comparing this joint probability $\pi_{11}^{H_{0}}(t)$ with the true probability of joint firing $\pi_{11}(t)$, one can test whether the increase of joint firing is due to an increase of synchronous action potentials. The null hypothesis, in terms of the probability of joint firing, is

$$
H_{0}: \tau(t)=\pi_{11}(t)-\pi_{11}^{H_{0}}(t)=0 .
$$

Using the delta method, the confidence bounds of $\tau(t)$ under the null are determined by

$$
\widehat{\tau}(t) \pm z_{1-\alpha / m} \sqrt{\widehat{\operatorname{Var}}(\widehat{\tau}(t))}
$$

and the variance of the joint probability of firing is estimated as

$$
\widehat{\operatorname{Var}}(\widehat{\tau}(t))=\left.\left(\frac{\partial \tau(t)}{\partial \boldsymbol{\beta}}\right)^{T} \widehat{\operatorname{Cov}}(\boldsymbol{\beta})\left(\frac{\partial \tau(t)}{\partial \boldsymbol{\beta}}\right)\right|_{\boldsymbol{\beta}=\hat{\boldsymbol{\beta}}},
$$

with $\widehat{\operatorname{Cov}}(\hat{\boldsymbol{\beta}})$ the estimated covariance matrix of $\hat{\boldsymbol{\beta}}$. At each time $t$, one can decide that there is a significant increase in synchrony if 0 lies out of the confidence bands. Since multiple comparisons are of interest in this situation (one test for each time point), the Bonferroni method can be used. Here, the use of $\alpha / m$ is recommended instead of $\alpha$, with $m$ the number of comparisons to be made. This test reveals excess - positive or negative- in synchrony provoked by the stimulus or any other behavioral condition. This is an interesting test which allows us to compare and quantify levels of synchronization between different situations at different moments, e.g., control (absence of stimulus) versus presence of stimulus. This methodology will be illustrated in the next section.

Note that other test can be of interest as well. One could be interested in testing whether the CSM stays equal to the 'independent' CSM, given that the 
marginal probabilities of firing change. The independent CSM has to be calculated under the assumption $\pi_{11}=\pi_{1+} \pi_{+1}$. This test removes the effect of the stimulus on synchronization. Therefore, this test complements the previous described because it compares the CSM based on the real data to CSM under an independent hypothesis. However, it could reveal correlation induced by changes in neurons firing rate but not due to synchrony.

\section{Data Analyses}

In neurophysiology, interest is in the temporal evolution of the firing rate and the synchrony between certain neurons, both being important neural mechanisms to transmit information about the characteristics of a stimulus. Further, one wants to investigate the effect of the stimulus properties (in this case, different orientations of the stimulus) on the firing rate and synchrony.

\subsection{Time Course}

As a first application of the model, we jointly investigate the temporal structure of spike trains and spike coincidences of neurons 1 and 2. Flexible models to describe the time trends are needed. For both the marginal probabilities as well as the synchrony, the model will be fit in a generalized linear models framework

$$
\begin{aligned}
\operatorname{logit}\left(\pi_{1+}(t)\right) & =f_{1}(t), \\
\operatorname{logit}\left(\pi_{+1}(t)\right) & =f_{2}(t), \\
\operatorname{logit}(\operatorname{CSM}(t)) & =f_{3}(t),
\end{aligned}
$$

with $f_{1}(t), f_{2}(t)$ and $f_{3}(t)$ some functions of time. Any flexible and parsimonious model for $f_{1}(t), f_{2}(t)$, and $f_{3}(t)$ could be chosen. We investigated both a parametric and a semi-parametric method: first a parametric polynomial with an orthonormal basis on $f_{1}(t), f_{2}(t)$ and $f_{3}(t)$ was chosen, then a semi-parametric piecewise cubic spline $f_{1}(t), f_{2}(t)$ and $f_{3}(t)$ was considered. As a model selection criterion, we 
Table 3: A description and comparison of models. The pseudo-likelihood value is displayed, together with the number of parameters in the model and the pseudolikelihood AIC value.

\begin{tabular}{llcccc} 
Model & Description & degree & $p \ell$ & $\#$ par & AIC \\
\hline 1 & orthonormal polynomials & 5 & -16661.13 & 18 & -16679.13 \\
2 & orthonormal polynomials & 10 & -16623.70 & 33 & -16656.70 \\
3 & piecewise-cubic splines & 5 & -16646.77 & 18 & -16664.77 \\
4 & piecewise-cubic splines & 10 & -16604.55 & 33 & -16637.55 \\
\hline
\end{tabular}

suggest to use the pseudo-likelihood $A I C=p \ell-r$, with $r$ the number of parameters in the model. Larger values of AIC indicate a better fitting model. A summary of model fit is given in Table 3 . In addition, pseudo-likelihood ratio tests comparing Model 2 with Model $1\left(G_{a}^{2}=72.08\right.$, p-value $\left.<0.0001\right)$ and comparing Model 4 with Model $3\left(G_{a}^{2}=93.47, \mathrm{p}\right.$-value $\left.<0.0001\right)$ are conducted. The piecewise-cubic spline of degree 10 seems to give the best fit. The estimated firing rate of a spike, i.e., the number of spikes per second, and the conditional synchrony measure, based on the piecewise cubic spline of degree 10, are shown in Figure 7 (solid line). The smoothed observed rates are represented by the dotted line. The natural spline model closely follows the observed rates, providing extra strength to believe the analysis.

Based on this model, several quantities of interest can be investigated. Both the firing rates in the two neurons, as well as the firing synchrony change during the discrimination task. Reactions on the stimulus are reflected in growing firing rates and increasing synchrony between the two neurons. After removal of the stimulus, the firing rates of both neurons and the synchrony drops back to the initial state. The maximal firing rate of neuron 1 appears $125 \mathrm{~ms}$ after the start of the stimulus, with a firing rate of 0.31 . The maximal firing rate of neuron 2 appears already at $100 \mathrm{~ms}$ after the start of the stimulus, with a firing rate of 0.27 . 

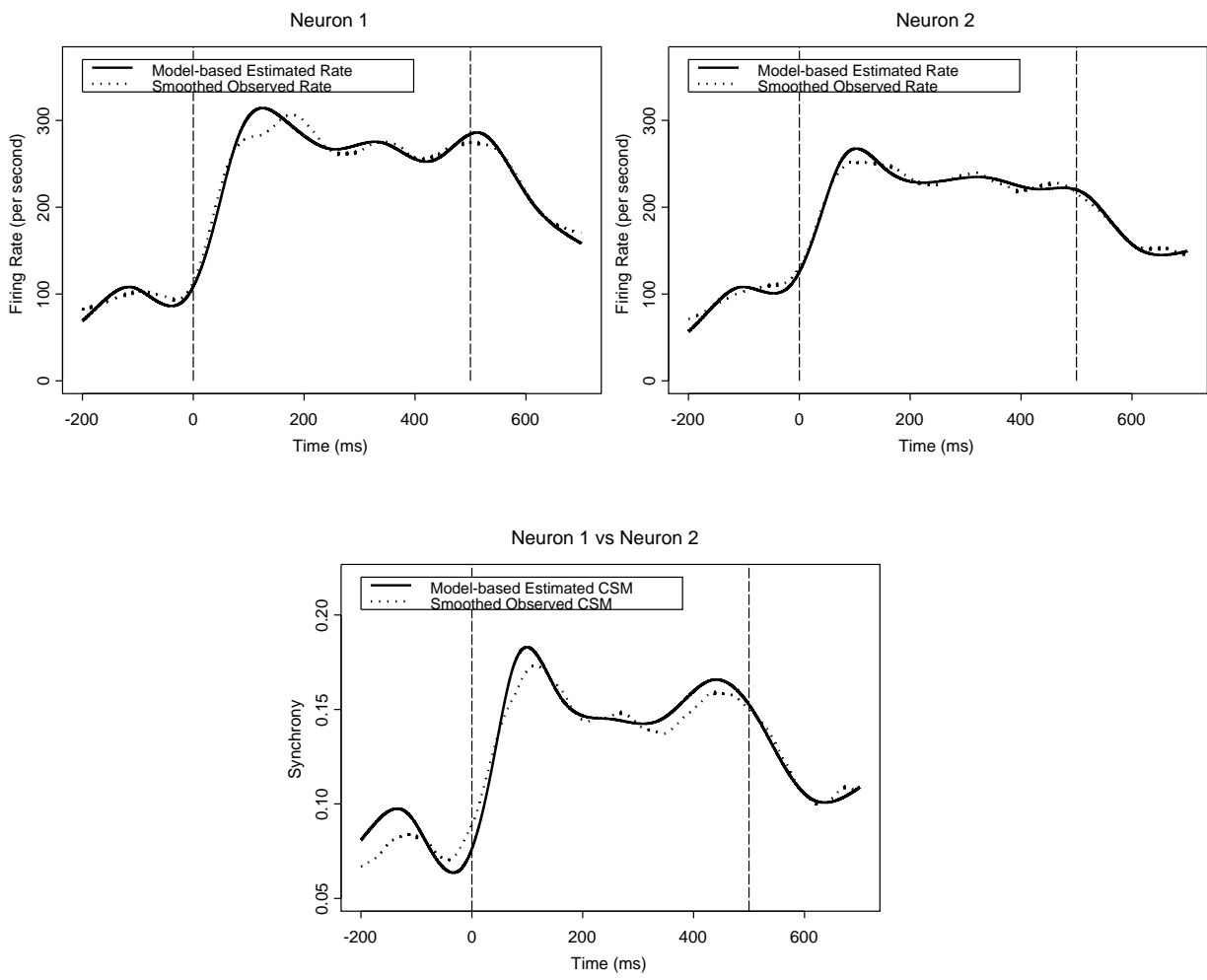

Figure 7: Model 1. The top panels present the estimated firing rate for neuron 1 and 2, respectively. The bottom panel presents the estimated synchrony between neurons 1 and 2.

To test whether the neurons are firing in synchrony, we use the test as proposed in Section 4.3. In the left panel of Figure 8, the joint firing rates (per second) under the null and alternative hypothesis are displayed. The full line is the estimated joint firing rate in the experiment. The dotted line represent the joint firing rate based on the baseline CSM. The joint probability of firing, under the null hypothesis of a constant conditional synchrony measure $\mathrm{CSM}=\mathrm{CSM}^{0}$ changes over time, due to the varying firing probabilities of the two neurons individually. To test for synchrony, we compare the true probability of joint firing with the null hypothesis of joint firing with a background CSM, as displayed in the right panel of Figure 8. The bold line is the estimated difference in the probability of joint firing. The $95 \%$ confidence 

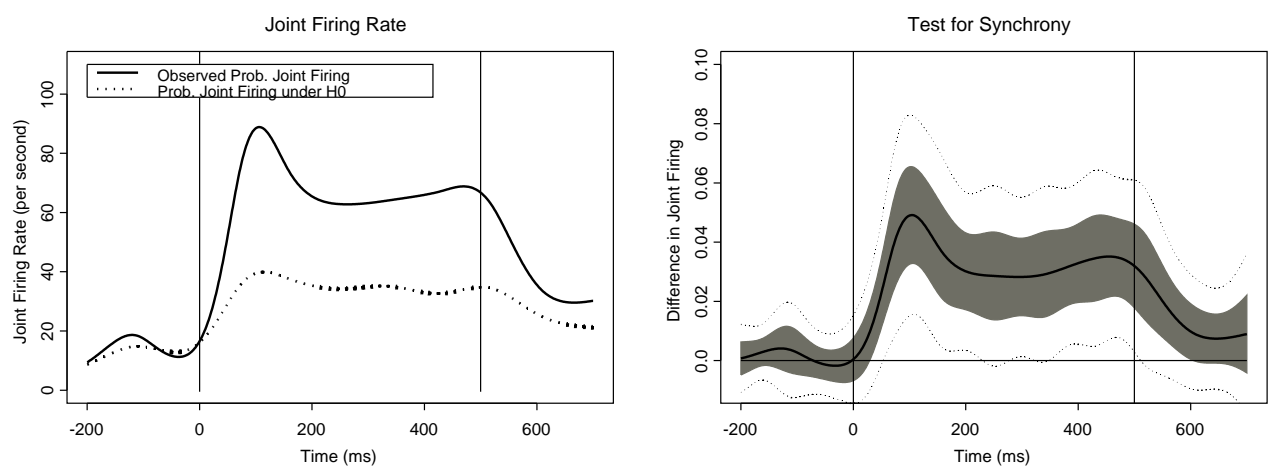

Figure 8: Test for synchrony. Left: test based on the conditional synchrony measure. Right: test based on the probability of joint firing.

bounds (grey band) and Bonferonni corrected confidence bounds (dotted lines) are displayed. By comparing the confidence bounds with 0 , one can see that there is a there is a significant increase of synchrony almost immediately after the stimulus is shown (after $53 \mathrm{~ms}$ ), which disappears again at $11 \mathrm{~ms}$ after removal of the stimulus. Note however that the Bonferroni method is highly conservative and may miss real differences, since the multiple tests are possibly highly correlated. Further, since the marginal probabilities and the association are freely varying, none of the methods developed for specific cases will apply (Hochberg and Tamhane, 1987). Since we believe that no off-the-shelf methods are available, we will consider both the corrected and uncorrected confidence bounds. This allows us to draw some first conclusions. However, the issue of multiple testing is important and needs more research.

\subsection{Orientation}

The orientation of the stimulus $\left(85^{\circ}, 90^{\circ}\right.$ or $\left.95^{\circ}\right)$ can have an important effect on the firing rate and on the synchronization of the neurons. One goal of this study is to quantitatively determine and formally compare how the temporal patterns of neuronal activity are affected by the different orientations of the stimuli. 

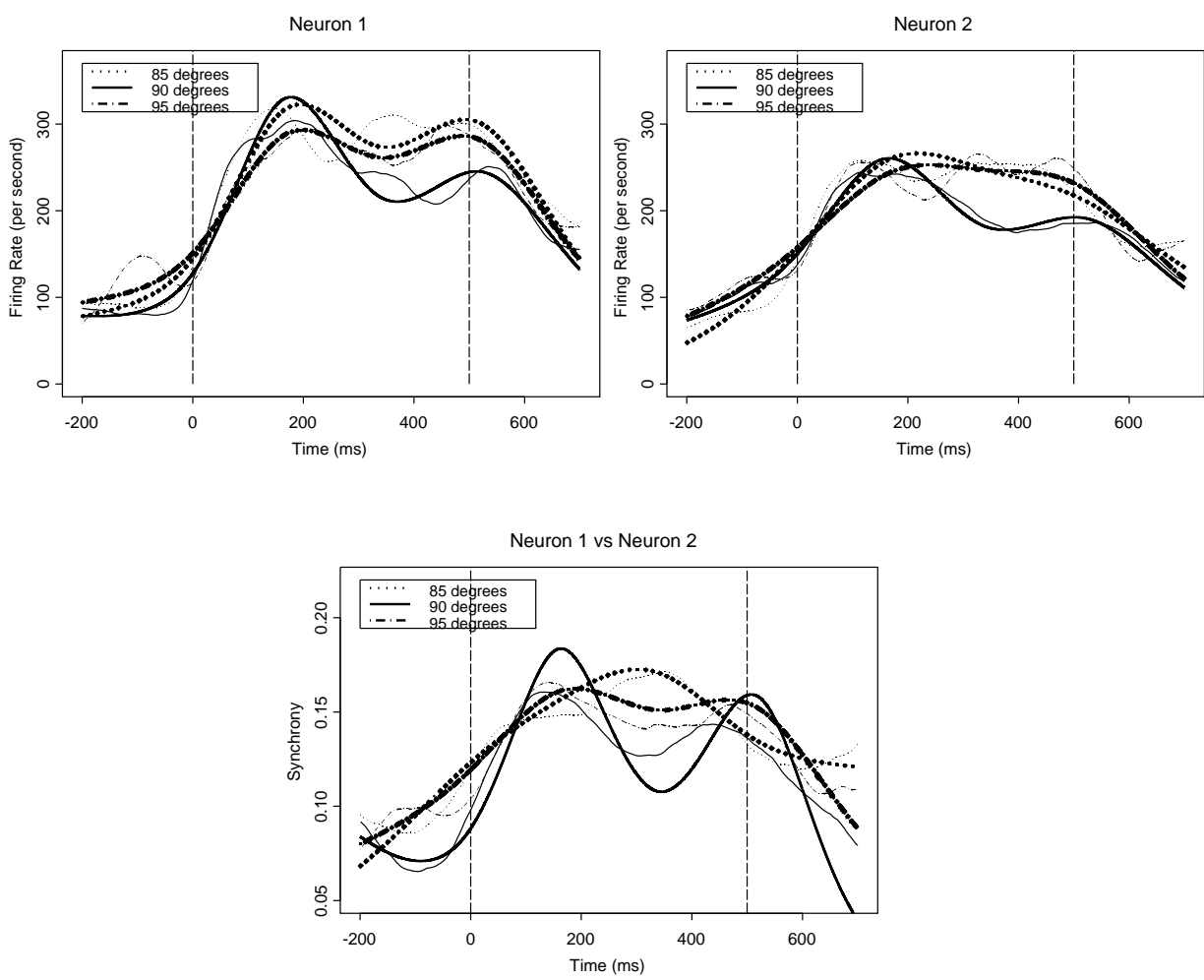

Figure 9: Model 2. The top panels present the estimated firing rate of neuron 1 and 2, respectively. The bottom panel presents the estimated synchrony between neurons 1 and 2.

Consider a model with inclusion of the orientation $\alpha$ :

$$
\begin{aligned}
f(t, \alpha)= & \left(\beta_{1}+\sum_{i=1}^{5} \beta_{1+i} f_{1}(t)\right)+\left(\beta_{7}+\sum_{i=1}^{5} \beta_{7+i} f_{2}(t)\right) I_{\alpha=85} \\
& +\left(\beta_{13}+\sum_{i=1}^{5} \beta_{13+i} f_{3}(t)\right) I_{\alpha=95}
\end{aligned}
$$

where $I_{\alpha=85}$ and $I_{\alpha=95}$ are dummy variables corresponding to orientations of $85^{\circ}$ and $95^{\circ}$, respectively, and $f_{1}(t), f_{2}(t)$, and $f_{3}(t)$ natural splines in time $t$ of degree 5. Results are presented in Figure 9. The solid line corresponds to $90^{\circ}$, the dotted line with $85^{\circ}$, and the dashed line with $95^{\circ}$. The firing rates in neurons 1 and 2 are slightly different for stimuli with orientations of $85^{\circ}$ or $95^{\circ}$, but they are quite 
Table 4: Pseudo-likelihood ratio test statistic $G_{a}^{2}$ and corresponding p-value for all pairwise comparisons on $\pi_{1+}, \pi_{+1}$, CSM and on the overall effect.

\begin{tabular}{crrr}
\hline Comparison & \multicolumn{1}{c}{$\pi_{1+}$} & $\pi_{+1}$ & \multicolumn{1}{c}{ CSM } \\
\hline $85^{\circ}=90^{\circ}$ & $25.58(<0.001)$ & $21.06(0.002)$ & $19.49(0.003)$ \\
$95^{\circ}=90^{\circ}$ & $17.08(0.009)$ & $20.03(0.003)$ & $7.76(0.256)$ \\
$85^{\circ}=95^{\circ}$ & $4.59(0.597)$ & $5.05(0.538)$ & $1.44(0.964)$ \\
$85^{\circ}=95^{\circ}=90^{\circ}$ & $32.04(0.001)$ & $31.13(0.002)$ & $16.36(0.175)$ \\
\hline
\end{tabular}

different for the $90^{\circ}$ oriented line. The thin lines represent the smoothed observed rates. This shows the model fits sufficiently well. In the primary visual cortex the receptive field position and its orientation preference changes across the visual space. For that reason, a long oriented lines, such as the ones used in the experiment, will provoke different responses in each cell. This explains the differences seen in the graph.

A pseudo-likelihood ratio test statistic, as proposed in Section 4.2, is calculated to test whether the effects of different orientations are different. Table 4 displays the pseudo-likelihood ratio test statistic $G_{a}^{2}$ and the corresponding p-value for the null hypothesis that the orientations have the same effect on $\pi_{1+}, \pi_{+1}$ and CSM separately, as well as for the overall effect. The effect of a $90^{\circ}$ stimulus on the firing rate of the neurons is significantly different from the effect of a $85^{\circ}$ or $95^{\circ}$ stimulus (all p-values $<0.001$ ). In contrast, there is no evidence for a difference between a $85^{\circ}$ or $95^{\circ}$ stimulus ( $\mathrm{p}$-value $>0.05$ ). The differences on the synchrony are less pronounced, with only a different effect between stimuli of $85^{\circ}$ and $90^{\circ}$.

In Figure 10, it is displayed whether there is a significant increase in the number of joint coincidences due to the stimulus, for each of the orientations separately. Based on the $95 \%$ confidence bound, we see that there is an immediate increase of joint firing when a stimulus of $85^{\circ}$ or $95^{\circ}$ is given. For a stimulus of $90^{\circ}$, the reaction is somewhat later in time. The difference between observed and expected 

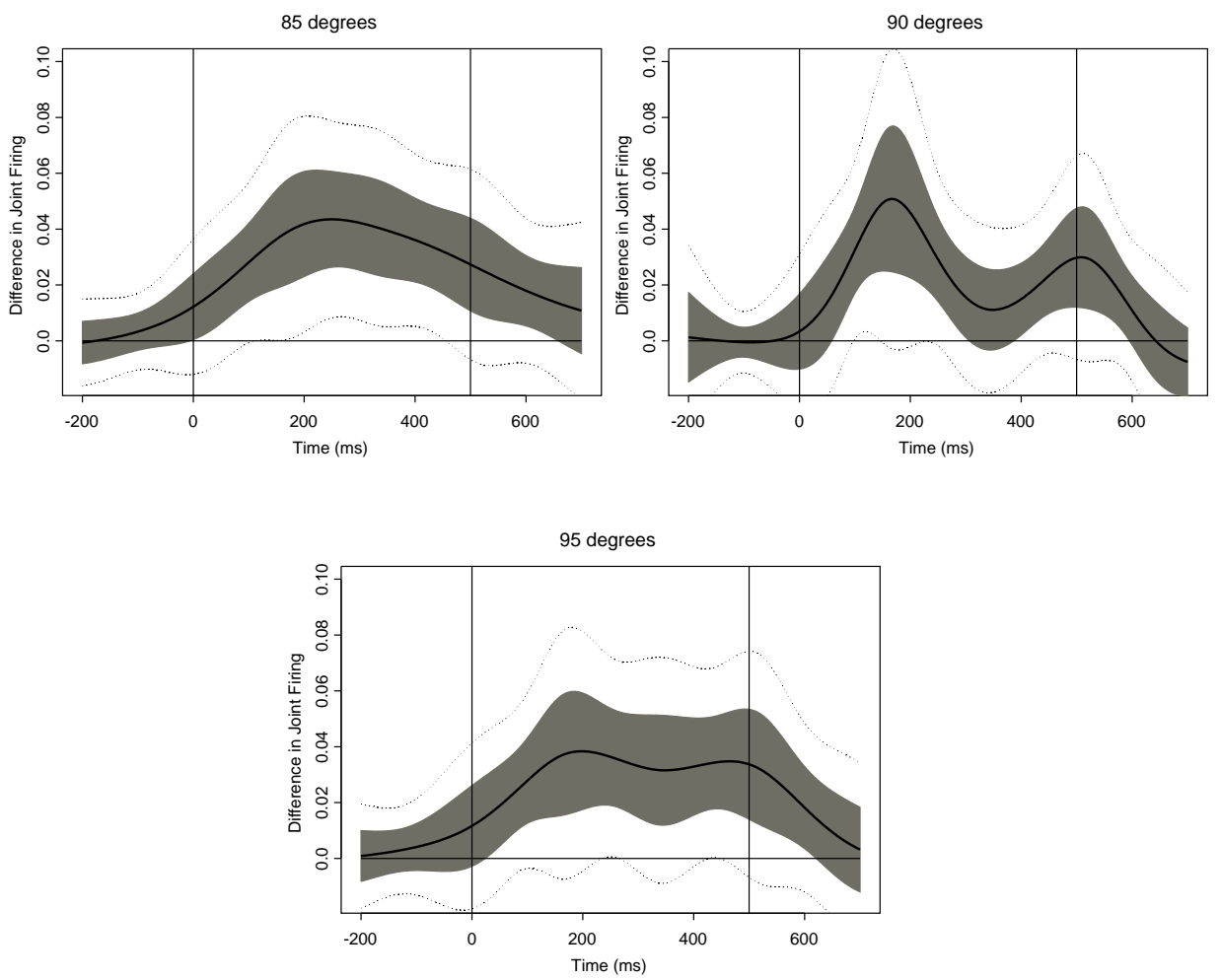

Figure 10: Test for synchrony, based on the probability of joint firing. From bottom to top: orientations 85, 90 or 95 degrees.

probability of synchronous spikes may be explained by postulating a common input or some other form of functional connectivity due to the stimulus. When we carry out the Bonferonni correction, a significant increase in joint firing is seen for a $85^{\circ}$ stimulus, whereas there is almost no evidence for a difference when a $90^{\circ}$ or $95^{\circ}$ stimulus is given. However, note that the power of the Bonferonni test is diminished due to the lack of independence between the tests, and thus possibly does not detect true differences in the population. 


\section{Discussion}

In this paper, we defined synchrony as the observation that action potentials emitted from different neurons are emitted at the same time. But, there is no "true" definition of synchrony, this term is dependent on the question and experimental situation. Synchrony can be defined at different temporal resolutions, e.g., 1, 3 and 5 ms. That is, spikes in one neuron were considered synchronous if they occurred at $0, \pm 1$, or $\pm 2 \mathrm{~ms}$, relative to spikes in the second neuron, respectively. The proposed conditional synchrony measure is flexible enough to allow extensions of the definition.

The conditional synchrony measure is the probability of firing together, given that at least one of the two neurons is active. The advantage of the CSM is that it is robust against the high number of $(0,0)$ matches, since these do not provide information about synchrony. While most association measures treat both $(0,0)$ and $(1,1)$ matches as synchronous events, the Conditional Synchrony Measure only uses the $(1,1)$ match as relevant synchronous event.

If one assumes that two spike trains have a certain background CSM, then the number of coincidences will depend on the firing rates of the neurons (this number will increase as the rates increase). Therefore, one cannot just compare the probability of joint firing with the baseline probability of joint firing, but one needs to include the firing rates of the neurons separately. By use of the CSM, one corrects for the probability that there is activity at a certain time point. Thus, comparing the CSM with the baseline value is a justifiable comparison. Our method allows the joint estimation of firing rates and synchrony measures adjusted by covariates. In this paper we have illustrated the model accounting for the effect of trial-specific covariate 'orientation'. This is a fixed covariate. This method is very flexible and allows also the introduction of time-varying covariates, such as the saccade (movement eyes).

Neurons of the visual cortex respond to oriented lines by modifying their firing 
rate, and the reaction to the stimulus depends on the position of the line over the receptive field of the cell. A long oriented line that falls on several receptive fields provokes different reactions. However, although each cell might respond differently and transmit different information, neighboring cells might synchronize their activity to such common stimulus and therefore constitute an assembly (Abeles 1982). CSM analysis of activity of two simultaneously recorded neurons reveals that the strength and temporal course of synchrony is modulated by the orientation of a common stimulus. This dynamic engagement of neural assemblies might transmit additional information about common features of a stimulus (Singer and Gray 1995, Shadlen and Movshon 1999).

The results shown here indicate that when the receptive fields of two simultaneously recorded neurons, close in visual space, are stimulated by long lines with different orientations, the response of each neuron varies (Figure 9, top panels). Changes in the relative position of each line $\left(85^{\circ}, 90^{\circ}, 95^{\circ}\right)$ over the receptive fields accounts for this variability. The level and time course of the synchrony provoked by the long lines varies according to the relative orientation of the stimulus over the neurons receptive fields (Figure 9, bottom panel; Table 4; Figure 10). This synchronization is associated with attentive perception of the orientation of the line stimulus, indicating that synchronization plays a functional role. In fact, monkeys cannot solve the task without paying attention to, and retain in working memory, the orientation of the line. These results agreed with others from the literature; synchrony between neurons depends on their relative receptive position in visual space (i.e., how far apart they are) and on orientation selectivity, and it its suggested to play a role in perception (Gray et al. 1989, Singer and Gray 1995, Vazquez et al. 1999, Kreiter and Singer 1996). In this regard our results confirmed previous ones, and therefore validate the statistic method we have developed. Our statistical approach will allow us to simultaneously compare synchronous activity of several neurons and to asses the effect of other behavioral events, such as eye movements, on synchrony (Martinez-Conde et al. 2000, Vazquez et al. 1999, Cano 2002). 
This is especially important due to the growing interest for statistical methods for analysis of multiple neural activities simultaneously recorded in electrophysiological preparations.

Although the method is presented in the specialized field of neurophysiology, the methodology is applicable in other medical and epidemiological areas where the similarity of a rare binary outcome among subjects is of interest (for example, twins studies). Thus, use of the proposed modelling approach is far beyond the neurophysiological context.

\section{References}

Abeles, M. (1982), Local cortical circuits. An electrophysiological study. Springer.

Abeles M, Prut Y, Bergman H, Vaadia E (1994), "Synchronization in neuronal transmission and its importance for information processing". Prog Brain Res, 102, 395-404.

Aerts, M., Geys, H., Molenberghs, G., and Ryan, L. (2002), Topics in Modelling of Clustered Data, London: CRC Press.

Aertsen, A.M., Gerstein, G.L., Habib, M.K., and Palm, G. (1989), "Dynamics of neuronal firing correlation: modulation of effective connectivity," J Neurophysiol, 61, 900-917.

Arnold, B.C., and Strauss, D. (1991), "Pseudolikelihood estimation : Some examples," Sankhya B, 53, 233-243.

Connolly, M.A. and Liang, K.Y. (1988), "Conditional logistic regression models for correlated binary data," Biometrika 75, 501-506.

Eggermont, J.J. (1998), "Is there a neural code?," Neurosci Biobehav Rev, 22, 355-370.

Ekholm, A., Smith, P. W. F., and McDonald, J. W. (1995), "Marginal regression analysis of a multivariate binary response," Biometrika, 82, 847-854. 
Gerstein, G.L., and Aertsen, A.M. (1985), "Representation of cooperative firing activity among simultaneously recorded neurons," J Neurophysiol, 54, 15131528.

Geys, H., Molenberghs, G. and Lipsitz, S.R. (1998), "A note on the comparison of pseudo-likelihood a nd generalized estimating equations for marginally specified odds ratio models," Journal of Statistical Computation and Simulation, 62, 45-72

Geys, H., Molenberghs, G. and Ryan, L. (1997), "Pseudo-likelihood inference for clustered binary data," Communications in Statistics: Theory and Methods, 26, 2743-2767.

Geys, H., Molenberghs, G., Ryan, L. (1999), "Pseudo-likelihood modelling of multivariate outcomes in developmental toxicology," Journal of the American Statistical Association ,94, 734-745.

Gray, C.M., Konig, P., Engel, A.K., Singer, W., (1989) "Oscillatory responses in cat visual cortex exhibit inter-columnar synchronization which reflects global stimulus properties," Nature, 338, 334-337.

Grün, S. (1996), "Unitary joint-events in multiple-neuron spiking activity: detection, significance, and interpretation," Reihe Physik, Band 60. Thun, Frankfurt/Main: Verlag Harri Deutsch.

Grün, S., Diesmann, M., Grammont, F., Riehle, A., and Aertsen, A. (1999), "Detecting unitary events without discretization of time," Journal of Neuroscience Methods, 94, 67-79.

Grün, S., Diesmann, M., and Aertsen, A. (2002a), "Unitary Events in Multiple Single-Neuron Spiking Activity: I. Detection and significance," Neural Computation, 14, 43-80.

Grün, S., Diesmann, M., and Aertsen, A. (2002b), "Unitary events in multiple single-neuron spiking activity: II. Nonstationary data," Neural Computation, $14,81-119$. 
Gütig, R., Aertsen, A., and Rotter, S. (2002), "Statistical significance of coincident spikes: count-based versus rate-based statistics," Neural Computation, 14, 121153.

Hernandez, A., Salinas, E., Garcia, R., Romo, R. (1997), "Discrimination in the sense of flutter: new psychophysical measurements in monkeys". J Neurosci, 17, 6391-6400.

Hochberg, Y. and Tamhane, A. C. (1987), Multiple Comparison Procedures, New York: John Wiley.

Hubel, D.H., Wiesel, T.N. (1969), "Anatomical demonstration of columns in the monkey striate cortex," Nature, 221, 747-750.

Hubel, D.H., Wiesel, T.N., Stryker, M.P. (1978), "Anatomical demonstration of orientation columns in macaque monkey," J Comp Neurol, 177, 361-380.

Johnson, R.A. and Wichern, D.W. (1998), Applied Multivariate Statistical Analysis. New Jersey: Prentice Hall.

Kass, R.E., Ventura, V., and Cai, C. (2003), "Statistical smoothing of neuronal data," Network, 14, 5-15.

Konig, P., Engel A.K., Singer, W. (1996), "Integrator or coincidence detector? The role of the cortical neuron revisited," Trends Neurosci, 19, 130-137.

Le Cessie, S. and Van Houwelingen, J.C. (1994), "Logistic regression for correlated binary data," Applied Statistics, 43, 95-108.

Liang, K.Y. and Zeger, S. (1986), "Longitudinal data analysis using generalized linear models," Biometrika 73, 13-22.

Malsburg, vdC. (1999), "The what and why of binding: the modeler's perspective," Neuron, 24, 95-25.

Martignon, L., Deco, G., Laskey, K., Diamond, M., Freiwald, W., and Vaadia, E. (2000), "Neural coding: higher-order temporal patterns in the neurostatistics of cell assemblies," Neural Computation, 12, 2621-2653. 
McCullagh, P. and Nelder, J.A. (1994), Generalized Linear Models. London: Chapman and Hall.

Mountcastle, V.B., Steinmetz, M.A., and Romo, R. (1990), "Frequency discrimination in the sense of flutter: psychophysical measurements correlated with postcentral events in behaving monkeys," J Neurosci, 10, 3032-3044.

Newsome, W.T., Britten, K.H., and Movshon, J.A. (1989), "Neuronal correlates of a perceptual decision," Nature, 341, 52-54.

Plackett, R.L. (1965), "A class of bivariate distributions," Journal of the American Statistical Association, 60, 516-522.

Riehle, A., Grün, S., Diesmann, M., and Aertsen, A. (1997), "Spike synchronization and rate modulation differentially involved in motor cortical function," Science 7, 278, 1950-1953.

Romo, R., and Salinas, E. (1999), "Sensing and deciding in the somatosensory system," Curr Opin Neurobiol, 9, 487-493.

Shadlen, M.N., and Movshon, J.A. (1999), "Synchrony unbound: a critical evaluation of the temporal binding hypothesis," Neuron, 24, 67-75.

Shadlen, M.N., and Newsome, W.T. (1994), "Noise, neural codes and cortical organization," Curr Opin Neurobiol, 4, 569-579.

Singer, W. (1999), "Neuronal synchrony: a versatile code for the definition of relations?" Neuron, 24, 49-25.

Singer W., Gray, C.M. (1995). Visual feature integration and the temporal correlation hypothesis. Annu Rev Neurosci 18: 555-586.

Usrey, W.M., Reid, R.C. (1999), "Synchronous activity in the visual system," Annu Rev Physiol, 61, 435-456.

Vazquez, P., Cano, M., Acuña, C. (2000), "Discrimination of line orientation in humans and monkeys," Journal of Neurophysiology, 83, 2639-2648. 


\section{Appendix}

The principal idea of the pseudo-likelihood methodology is to replace the numerically challenging joint density by a simpler function. The method is well described by Arnold and Strauss (1991), and also found in Conolly and Liang (1988), Liang and Zeger (1986) and Le Cessie and Van Houwelingen (1994). While the method achieves important computational economies by changing the method of estimation, it provides consistent and asymptotical normal estimates (Arnold and Strauss 1991) and it does not affect model interpretation.

Let $\boldsymbol{Y}_{i}$ be the vector of binary outcomes for subject $i(i=1, \ldots, N)$. Without loss of generality we can assume that $\boldsymbol{Y}_{i}$ has constant dimension $L$. The extension to variable lengths of $\boldsymbol{Y}_{i}$ is straightforward. Define $S$ as the set of all $2^{L}-1$ vectors of length $M$, consisting solely of zeros and ones, with each vector having at least one nonzero entry. Denote by $\boldsymbol{y}_{k}^{(s)}$ the subvector of $\boldsymbol{y}_{k}$ corresponding to the components of $s \in S$ that are nonzero. The associated joint density is written as $f_{s}\left(\boldsymbol{y}_{k}^{(s)} ; \boldsymbol{\Theta}_{k}\right)$, with $\boldsymbol{\Theta}_{k}=\boldsymbol{X}_{k} \boldsymbol{\beta}$. Specify a set $\delta=\left\{\delta_{s} \mid s \in S\right\}$ of $2^{L}-1$ real numbers, with at least one nonzero component and define the log pseudo-likelihood as:

$$
p \ell=\sum_{i=1}^{N} \sum_{s \in S} \delta_{s} \ln f_{s}\left(\boldsymbol{y}_{i}^{(s)} ; \boldsymbol{\Theta}_{i}\right),
$$

where some (thought not all) of the $\delta_{s}$ 's may be negative. This must correspond to a product of marginal and conditional densities.

One example of a possible pseudo-likelihood function is to replace the full likelihood function for trial $j(j=1, \ldots, N)$, i.e., $f_{j}\left(y_{11 j}, \ldots, y_{1 T j}, y_{21 j}, \ldots, y_{2 T j}\right)$ by

$$
p \ell_{j}=\sum_{j=1}^{N} \ln g\left(y_{1 t j}, y_{2 t j}\right),
$$

where the index $j$ indicates the outcomes of trial $j$. In this setting the pseudolikelihood definition is completed using equations 4.4. The value of the parameters 
that maximize the log pseudo-likelihood function $p \ell=\sum_{j=1}^{N} p \ell_{j}$ are the pseudolikelihood estimates.

Arnold and Strauss (1991) established consistency and asymptotic normality of the pseudo-likelihood estimator. Similar in spirit to generalized estimating equations (Liang and Zeger 1986), the asymptotic normality result provides an easy way to consistently estimate the asymptotic covariance matrix:

$\widehat{\operatorname{Cov}}(\hat{\beta})=J^{-1} K J^{-1}=\left.\left(\sum_{j=1}^{N} \frac{\partial U_{j}}{\partial \beta}\right)^{-1}\left(\sum_{j=1}^{N} U_{j}(\beta) U_{j}(\beta)^{T}\right)\left(\sum_{j=1}^{N} \frac{\partial U_{j}}{\partial \beta}\right)^{-1}\right|_{\beta=\hat{\beta}}$

with $U_{j}$ the pseudo-likelihood estimating equations

$$
U_{j}(\beta)=\frac{\partial \ln g\left(y_{1 t}, y_{2 t}\right)}{\partial \beta} .
$$

This approach acknowledges the fact that, while the synchrony between different neurons on the same time point is often of scientific interest, the association between different time points is usually considered a nuisance. The sandwich variance estimator (6.3) is then used to adjust for potential bias in the variance estimator.

An important advantage of the pseudo-likelihood approach is the close connection with likelihood, which enabled Geys, Molenberghs and Ryan (1999) to construct pseudo-likelihood ratio test statistics that have easy-to-compute expressions and intuitively appealing limiting distributions. We restrict attention to the pseudolikelihood ratio test statistic. Suppose we are interested in testing the null hypothesis $H_{0}: \gamma=\gamma_{0}$, where $\gamma$ is an $r$-dimensional subvector of the $p$-dimensional vector of regression parameters $\boldsymbol{\beta}$ and write $\boldsymbol{\beta}$ as $\left(\boldsymbol{\gamma}^{T}, \boldsymbol{\delta}^{T}\right)^{T}$. Then, the pseudo-likelihood ratio test statistic, defined by

$$
G_{a}^{* 2}=2\left[p \ell\left(\widehat{\boldsymbol{\beta}}_{N}\right)-p \ell\left(\boldsymbol{\gamma}_{0}, \widehat{\boldsymbol{\delta}}\left(\boldsymbol{\gamma}_{0}\right)\right)\right] / \overline{\boldsymbol{\lambda}},
$$

is approximately $\chi_{r}^{2}$ distributed. In this definition, $\widehat{\boldsymbol{\beta}}_{N}$ is the pseudo-likelihood parameter estimate of $\boldsymbol{\beta}$ and $\widehat{\boldsymbol{\delta}}\left(\boldsymbol{\gamma}_{0}\right)$ denotes the maximum pseudo-likelihood estimator in the subspace where $\gamma=\gamma_{0}$. Further, $\bar{\lambda}$ is the mean of the eigenvalues of $\left(J^{\gamma \gamma}\right)^{-1} \Sigma_{\gamma \gamma}$, where $J^{\gamma \gamma}$ is the $r \times r$ submatrix of the inverse of $J$ and $\Sigma_{\gamma \gamma}$ is the submatrix of $\Sigma=J^{-1} K J^{-1}$. 